\title{
Identification of dietary patterns associated with characteristics of successful aging
}

\author{
Nik Nur Izzati Nik Mohd Fakhruddin ${ }^{1}$, Suzana Shahar ${ }^{1, *}$, Roslee Rajikan ${ }^{2}$, Mohd \\ Azahadi Omar ${ }^{3}$, Normah Che Din ${ }^{4}$, Rosdinom Razali ${ }^{5}$, Sakinah Harith ${ }^{6}$, Hamid \\ Jan Jan Mohamed ${ }^{7}$ \& Bibi Nabihah Abdul Hakim ${ }^{1}$
}

${ }^{1}$ Centre of Healthy Aging and Wellness, Faculty of Health Sciences, Universiti Kebangsaan Malaysia, Jalan Raja Muda Abdul Aziz, 50300 Kuala Lumpur, Malaysia; ${ }^{2}$ Dietetics Programme, School of Healthcare Sciences, Faculty of Health Sciences, Universiti Kebangsaan Malaysia, Jalan Raja Muda Abd Aziz, 50300 Kuala Lumpur, Malaysia; ${ }^{3}$ Centre for Burden of Disease Research, Institut for Public Health, Jalan Setia Murni U13/52, Alam Nusantara, 40170, Shah Alam, Malaysia; ${ }^{4}$ Health Psychology Programme, School of Healthcare Sciences, Faculty of Health Sciences, Universiti Kebangsaan Malaysia, Jalan Raja Muda Abd Aziz, 50300 Kuala Lumpur, Malaysia; ${ }^{5}$ Department of Psychiatry, Universiti Kebangsaan Malaysia Medical Centre, Jalan Yaacob Latif, Bandar Tun Razak, Cheras, 56000 Kuala Lumpur, Malaysia; ${ }^{6}$ School of Nutrition and Dietetics, Faculty of Health Sciences, Universiti Sultan Zainal Abidin, 21300 Kuala Terengganu, Terengganu, Malaysia; ${ }^{7}$ Nutrition and Dietetics Programme, School of Health Sciences, Universiti Sains Malaysia, 16150 Kubang Kerian, Kelantan, Malaysia

\begin{abstract}
Introduction: This study aimed to identify dietary patterns (DPs) and their association with successful aging (SA). Methods: A comparative cross-sectional study was conducted among 579 elderly subjects recruited from four states in Malaysia through a multistage random sampling method. SA was defined as having no chronic illnesses, no functional limitation, normal global function, no depression, a good quality of life and good self-perceived health. Information on dietary intake was obtained using a diet history questionnaire. Cognitive functions were assessed using the Mini Mental State Examination (MMSE), depressive symptoms using Geriatric Depression Score-15 items (GDS-15) and a question regarding their perceived health and quality of life. Activities of Daily Living (ADL) and Instrumental Activities of Daily Living (IADL) questionnaires were used to determine functional status. DPs were obtained using the principal component analysis (PCA) approach. An ordinal logistic regression model was used to examine associations between DP scores and SA. Results: Five DPs were identified namely 'sweet foods-beverages', 'meat-vegetablesrice and noodles', 'local snacks-fish and seafood-high salt foods', 'fruits-legumes', and 'tropical fruits-oats'. A higher score for 'tropical fruits-oats' DP was associated with SA [Adjusted OR=1.59 (95\% CI: 1.08-2.32)]. However, the association diminished when the model was adjusted for education level. Further analysis indicated that this DP increased the chance of SA among those with secondary education and above [Adjusted OR=2.43 (95\% CI: 1.09-5.42)]. Conclusion: 'Tropical fruits-oats' DP is associated with SA among elderly with secondary education and above. There is a need to investigate DPs among those with lower education.
\end{abstract}

Keywords: Cognitive function, dietary pattern, elderly, principal component analysis, successful aging

\footnotetext{
*Corresponding author: Prof. Dr Suzana Shahar

Centre of Healthy Aging and Wellness, Faculty of Health Sciences, Universiti Kebangsaan Malaysia, Jalan Raja Muda Abdul Aziz, 50300 Kuala Lumpur, Malaysia

Tel: +(6)03-9289 7163/8066; Fax: +(6)03-2691 4304; E-mail: suzana.shahar@ukm.edu.my doi: https://doi.org/10.31246/nutriweb-2018-0052
} 


\section{INTRODUCTION}

Successful aging (SA) ranges widely across the social, psychosocial and the medical sciences fields. It includes consideration of life satisfaction, wellbeing, the maintenance of cognitive and physical functions, as well as good mental and physical health (Bowling \& Dieppe, 2005). Mild cognitive impairment (MCI) is a reversible condition between the normal and dementia state (Petersen et al., 2014). Vanoh et al. (2017) have outlined a set of criteria for usual aging (UA) that includes having an average cognitive function, with no dementia, no physical limitation, but being at a higher risk of having major chronic diseases. Traditionally, food intakes with a wide range of nutrients have been studied for their role in health. However, since foods are eaten together, there is a growing interest in determining dietary patterns and their association with health, rather than a single nutrient. The original aim of a dietary pattern (DP) analysis was to capture the overall diet (Imamura \& Jacques, 2011). A DP, which is a summary of an eating style, is able to increase the awareness of healthy eating by promoting suitable food choices (Freeland-Graves \& Nitzke, 2002). The interest in DPs and cognitive functions has been growing rapidly because a DP is an alternative approach to addressing the complexity of the human diet and the synergistic effects of different foods and nutrients on the health of individuals.

There are two main approaches in deriving DPs. These are the a priori and the a posteriori methods (Hu, 2002). In the a priori method, a DP is created based on the knowledge about 'healthy' diet, dietary guidelines or predefined dietary scores for a specific DP, including the Mediterranean Diet and Dietary Approach to Stop Hypertension (DASH) Diet (Hu, 2002). A review by RomanViñas and Serra-Majem (2014) indicated a reduction in the overall mortality, incidence of cardiovascular disease, and functional capacity for individuals older than 65 years who followed a healthy lifestyle. Furthermore, compliance with such a DP, increased the chances for aging successfully by $58 \%$ after 10 years (Gopinath et al. 2016). On the other hand, the a posteriori method involved modelling dietary data through factor analysis, cluster analysis or reduced rank regression to derive a particular DP (Hu, 2002). A posteriori DPs or datadriven DPs do not require any hypothesis about the beneficial or detrimental effects of specific foods on health. So far little was known about specific DPs that prudently promoted SA and reduced risk of cognitive impairment among Asians, except for a study by Chan, Chan $\&$ Woo (2013) which was conducted among older Chinese adults in Hong Kong. However, this study did not use a comprehensive neuropsychological assessment method for cognitive functions and the subjects had a higher educational level.

The aim of this study was to identify a DP associated with SA among multiethnic elderly persons in Malaysia, using the a posteriori method. This study was part of a larger study designed for development of a neuro-protective model for healthy longevity named LRGS TUA, as published elsewhere (Fakhruddin et al., 2016; Shahar et al., 2016).

\section{MATERIALS AND METHODS}

\section{Study design and subjects}

A secondary analysis involving 579 out of 2,322 subjects from LRGS TUA study baseline database was conducted. LRGS TUA involved multistage random sampling of elderly from four states of Peninsular Malaysia, which had the highest numbers of elderly. These were Selangor, Johor, Kelantan and Perak which are approximately located at the centre, south, east and north of Peninsular Malaysia, respectively. The lists of elderly in these areas were given by the Department of Statistics, Malaysia. The study was conducted between May 
2013 and February 2014. The inclusion criteria were Malaysian citizenship, aged $\geq 60$ years. Those who were bedridden, had serious physical disabilities, mental problems such as depression and dementia, as well as those who were mute and deaf, were excluded from this study. In this secondary analysis, subjects from the primary analysis who had completed dietary assessment and classified as SA, UA or MCI aging groups and matched for age ( \pm 10 years), race and sex were included as samples. This project was approved by the Research Ethics Committee of National University of Malaysia (UKM 1.5.3.5/244/NN-0602013). Written consent was obtained from all participants before the interview session started.

\section{Data collection}

Information on sex, race, religion, age, marital status, education, living status, anthropometry, medical profile and dietary intake of the subjects was obtained through face-to-face interviews conducted at the respective community centres using a pre-tested questionnaire. The cognitive status of the subjects was neuropsychologically assessed. Their functional and psychosocial status, including depression, quality of life and self-perceived health status, were also measured.

\section{Criteria of successful aging}

All the aging subjects were classified into the three groups, namely SA, UA and MCI, based on the tests described by Shahar et al., (2016). The tests for classification into the three groups included the Activity of Daily Living (ADL) (Katz et al., 1970), Instrumental Activity of Daily Living (IADL) (Lawton \& Brody, 1969), Mini Mental State Examination (MMSE) (Razali et al., 2014), Geriatric Depression Symptoms-15 items (GDS15) (Yesavage et al., 1982), Rey Auditory Verbal Learning Test (RAVLT) (Schmidt, 1996), Digit Span (Wechsler, 1958) and two psychosocial questions that measured the quality of life and selfperceived health. The ADL test was used to assess the ability of the subjects to perform personal activities including bathing, dressing, eating, transferring and toileting. The IADL test involved the assessment of more complex activities including shopping, walking 200 metres, going around by car or using public transport by themselves, going up the stairs or doing heavy work around the house.

SA subjects were categorised based on six main criteria including (1) the absence of chronic diseases such as diabetes mellitus, hypertension, cancer, heart diseases, chronic lung disease and stroke, (2) no functional limitations, (3) normal global function, (4) no depression, (5) good quality of life and (6) self-perceived good health. Any participants who had MMSE score of $>14$ and did not meet any criteria of SA and MCI were categorised into the UA group.

In addition, MMSE was used as a tool to assess global cognitive functions with cut-off $\geq 22$ to indicate SA and $\geq 19$ for MCI. Depression was assessed using the GDS-15. Those with scores of $>5$ were excluded from this study as this indicated depression. Question 10 on the GDS-15 was used to measure subjective memory problems of the respondents. Subjective memory complaints were present if the subject answered 'Yes'. The quality of life was assessed by asking the subjects: 'In general, how do you perceive your quality of life?' Finally, self-perceived health was assessed using the single question: 'How would you describe your health status?' The answers to both these questions were rated using Likert scales. Subjects who were suspected of having dementia were referred to and further assessed by a psychiatrist.

\section{Food intake}

Information on food intake of the respondents was collected using the validated Diet History Questionnaire 
Table 1. Food group classification

\begin{tabular}{ll}
\hline Food groups & Food items \\
\hline Rice and noodles & Rice porridge, white rice, dried noodle and wet noodle \\
Oats & Oats \\
Flatbread & Roti canai \\
Meats & Beef and pork \\
Fish and seafoods & Pomfret, tuna, Indian mackerel, round scad, red snapper, hairtail \\
& scad, trevally, sea perch, Spanish mackerel, cuttlefish and prawn \\
Dairy products & Full cream milk and butter \\
Legumes & Soy bean fermented cake, soy bean cake and yellow dhal \\
Tropical fruits & Papaya, orange, banana, duku and rambutan \\
Fruits & Red apple, green apple and grapes \\
Vegetables & Brinjal, okra, carrot, cucumber, broccoli, long bean, chilli, spinach, \\
& mustard leaves, tapioca shoots, ulam raja ${ }^{\ddagger}$, swamp cabbage, fern \\
shoots, mung bean, cabbage and kale \\
High salt foods & Banana fritters, cucur ${ }^{\S}$, banana ball, curry puff, and cakoi ${ }^{\pi}$ \\
Sweet foods & Salted fish, anchovy sauce, fermented durian and chilli shrimp paste \\
Beverages & Sweetened creamer, granulated sugar, egg jam and fruit jam \\
\hline
\end{tabular}

${ }^{\dagger}$ Flatbread composed of dough containing copious amounts of fat (ghee-clarified butter), egg, flour and water

${ }^{\ddagger}$ Malay traditional salad

${ }^{\S}$ Fried bite-sized snack usually made from flour and anchovies

"Long golden-brown deep-fried strip of dough commonly served with egg jam

(DHQ) of Fakhruddin et al. (2016). The subjects or caregivers were required to recall all foods and drinks that were consumed daily to obtain information on the usual dietary habits of the subject in a week. During the interview, the subjects were asked specific questions regarding the meals that they took such as type, portion size, frequency, time and place the foods were consumed. Portion size was estimated using household measurements such as a cup, a bowl, a spoon and a plate. Description of the cooking methods and home recipes were recorded as well. More than 350 types of food were reported by the subjects and were extracted from the DHQ. Food items were classified into 14 groups based upon their similarities or references from other studies (Table 1). There were some individual food items such as roti canai and oats being analysed individually either because it was inappropriate to incorporate them into a certain food group or because they were believed to represent a distinct DP. The evaluation of misreporting energy intake, i.e. the under and over-reporting of energy intake, was conducted during the primary analysis (Fakhruddin et al., 2016). The under-reporters were excluded.

\section{Statistical analysis}

Statistical analysis was performed using the Statistical Package for the Social Sciences (SPSS) IBM 21 to derive DPs on the basis of the original food group variables (consumption in g/day). The Bartlett test of sphericity (BTS) $(p<0.05)$ and the Kaiser-Meyer-Olkin (KMO) measure of sampling adequacy $(>0.50)$ were initially used to evaluate whether the data was suitable for a principal component analysis (PCA). The PCA method was used for the factor extraction, followed by a varimax rotation to assist in the interpretation of the results and to ensure that the factors were not correlated. Eigenvalues 
$>1$ were used to determine the number of DPs that were retained. In this study, five main components were extracted, which accounted for $47.5 \%$ of the total variances. Specific DPs were labelled on the basis of the types of food exhibiting the strongest correlation and having the highest loading factors. Positive loading factors indicated that the food groups were positively correlated with the DP and negative values indicated that the food groups were inversely correlated with the DP. The food groups were retained in the DP if the factor loading value was $\geq 0.3$ (Chan et al., 2013). For each subject, the individual DP score was calculated by adding the intake of 14 food groups weighted by their factor loading. The factor scores that were obtained for each factor defined the position of each individual along a gradient. These were then ranked as tertile groups to indicate a low, medium or high intake, which indicated the degree to which the individual's diet conformed to the DP. The highest tertile was then compared with the lowest tertile of factor scores for each of the DP. Ordinal logistic regression $(\mathrm{MCI}=0, \mathrm{UA}=1, \mathrm{SA}=2)$ was used to assess the association between the DPs and SA, with adjustments of age, gender, race, calorie intake, body mass index (BMI), marital status and smoking status as covariates. Further analyses were done using two models i.e. model 1 , without educational level, and model 2 with educational level, in addition to other covariates mentioned above.

\section{RESULTS}

\section{Dietary patterns}

The flow-chart of the process for the identification of DP associated with SA is shown in Figure 1. PCA identified five DPs with eigenvalues $>1$. The factor loadings of specific food items on each of the five identified DPs are shown in Table 2. A 'sweet foods-beverages' pattern was defined by high loading of sweet foods including sugar, condensed milk, sweet spread and beverages such as tea, coffee and malted drink, as well as low intake of dairy products including low fat milk and butter. A 'meats-vegetablesrice and noodles' pattern consisted of high intake of meat including beef and pork, various types of vegetables and also rice and noodles. A local snacksfish and seafood-high salt foods' pattern was defined as high consumption of Malaysian snacks, fish and seafood, sauces and condiments. Another DP was 'fruits-legumes', which was defined by a high intake of a wide range of fruits other than tropical fruits, and also legumes and legume-based products. The last DP was 'tropical fruits-oats', defined as high loadings of a wide range of tropical fruits that included papaya, orange, duku, banana and rambutan and oats.

351 types of food items extracted from DHQ

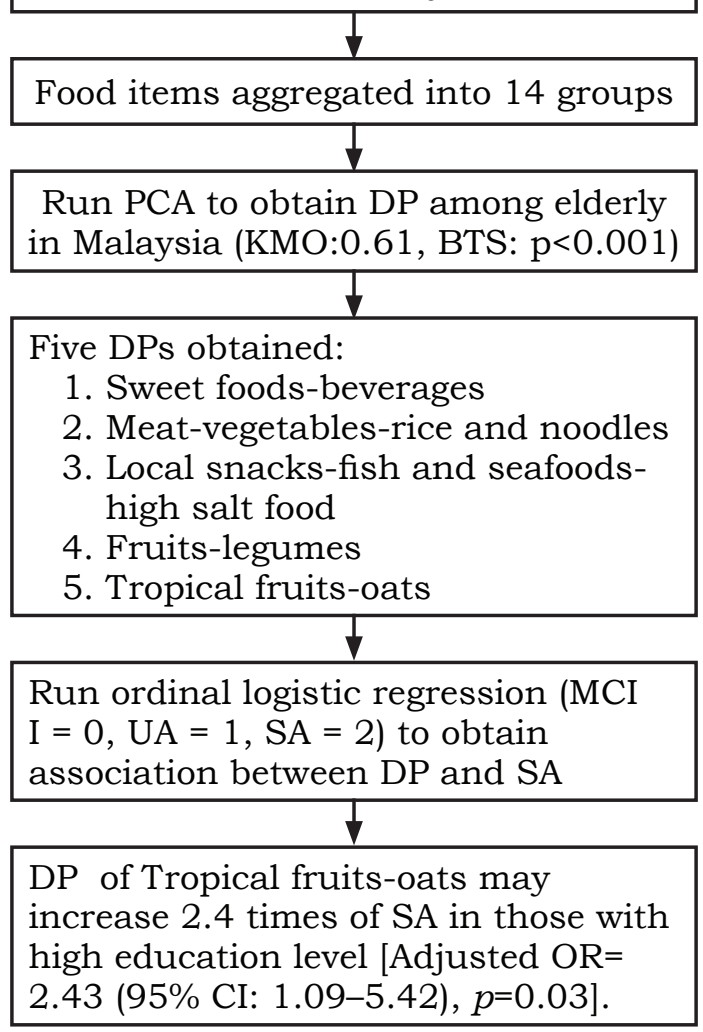

Figure 1. Flow chart of identification dietary patterns for SA 
Table 2. Factor-loading matrix for five DPs identified by PCA $(n=579)^{\dagger}$

\begin{tabular}{|c|c|c|c|c|c|}
\hline Food groups & $\begin{array}{c}\text { Sweet } \\
\text { foods- } \\
\text { beverages }\end{array}$ & $\begin{array}{l}\text { Meat- } \\
\text { vegetables- } \\
\text { rice and } \\
\text { noodles }\end{array}$ & $\begin{array}{l}\text { Local snacks- } \\
\text { fish and } \\
\text { seafoods- } \\
\text { high-salt food }\end{array}$ & $\begin{array}{l}\text { Fruits- } \\
\text { legumes }\end{array}$ & $\begin{array}{l}\text { Tropical } \\
\text { fruits-oats }\end{array}$ \\
\hline Beverages & 0.65 & 0.08 & 0.11 & 0.08 & 0.02 \\
\hline Sweet foods & 0.64 & 0.04 & 0.22 & 0.32 & 0.04 \\
\hline Dairy products & -0.62 & 0.03 & 0.39 & 0.24 & 0.01 \\
\hline Vegetables & -0.01 & 0.65 & 0.10 & -0.06 & 0.08 \\
\hline Rice and noodles & 0.13 & 0.58 & -0.10 & -0.17 & -0.24 \\
\hline Meats & 0.02 & 0.56 & -0.25 & 0.30 & 0.12 \\
\hline Local snacks & 0.02 & -0.21 & 0.64 & 0.11 & 0.09 \\
\hline Fish and seafoods & 0.31 & 0.08 & 0.58 & -0.19 & -0.26 \\
\hline High salt foods & 0.20 & 0.18 & 0.53 & 0.34 & -0.06 \\
\hline Legumes & 0.00 & 0.09 & 0.04 & 0.58 & 0.06 \\
\hline Fruits & -0.18 & 0.07 & 0.38 & 0.43 & 0.19 \\
\hline Roti canai & 0.20 & -0.28 & 0.03 & -0.41 & 0.28 \\
\hline Tropical fruits & 0.10 & -0.04 & -0.01 & -0.20 & 0.72 \\
\hline Oats & -0.34 & 0.32 & -0.05 & -0.06 & 0.51 \\
\hline Total variance explained (\%) & 11.7 & 9.6 & 9.3 & 9.2 & 8.2 \\
\hline $\begin{array}{l}\text { Cumulative variance } \\
\text { explained }(\%)\end{array}$ & 11.7 & 20.8 & 30.1 & 39.3 & 47.5 \\
\hline
\end{tabular}

DP, Dietary pattern

${ }^{\dagger}$ Factor loadings with absolute value $|>0.3|$ are shown in bold. For food group loads more than one dietary pattern, only the highest absolute value of loading is bolded.

\section{Overall association between DPs and SA}

According to Table 3, after accounting for various confounders such as BMI, calorie intake, smoking status and marital status, there was a positive association across the tertiles of 'tropical fruits-oats' DP and SA [Adjusted OR= 1.59 (95\% CI: 1.08-2.32), $p=0.02]$. After the addition of education level, the association for this DP across the tertile groups was diminished [Adjusted OR=1.30 (95\% CI: 0.87-1.93), $p=0.19]$. Thus, further analyses were conducted according to educational level stratification which are low and high education. Subjects who had no formal education or only attended primary school were categorised as 'low education' group. Subjects who attended secondary school or college were classified as 'high education' group. It was found that 'tropical fruitsoats' DP increased chances of SA only among those with education background of secondary school and college level [Adjusted OR= 2.43 (95\% CI: 1.09-5.42), $p=0.03$ ] (table not shown).

\section{Characteristics of subjects with successful aging DP}

Table 4 shows the characteristics of the subjects by tertile of 'tropical fruits-oats' DP. There was a significant difference between smoking status, calorie intake and education level between tertile groups of 'tropical fruits-oats' DP. Percentage of smoking decreased, whilst non-smoking increased in higher tertile groups. Calorie intake was significantly higher in T1 group as compared to T2 and T3 groups. However, there was no significant difference in calorie intake between T2 and T3 groups. In terms of educational level, subjects in T3 group obtained higher education level as compared to $\mathrm{T} 2$ and $\mathrm{T} 1$ groups. 
Table 3. Ordinal regression models of DP and SA in tertiles

\begin{tabular}{|c|c|c|}
\hline \multirow{2}{*}{$\begin{array}{l}\text { Dietary patterns } \\
\text { (Tertiles) }\end{array}$} & Model $1^{\ddagger}$ & Model $2 \S$ \\
\hline & AOR (95\% CI) & AOR (95\% CI) \\
\hline \multicolumn{3}{|c|}{ Sweet foods-beverages } \\
\hline $\mathrm{T} 1^{\dagger}$ & 1.00 & 1.00 \\
\hline $\mathrm{T} 2$ & $1.14(0.79-1.66)$ & $1.26(0.86-1.86)$ \\
\hline T3 & $1.15(0.77-1.72)$ & $1.18(0.78-1.78)$ \\
\hline$P$ trend & 0.48 & 0.41 \\
\hline \multicolumn{3}{|c|}{ Meat-vegetables-rice and noodles } \\
\hline $\mathrm{T} 1^{\dagger}$ & 1.00 & 1.00 \\
\hline $\mathrm{T} 2$ & $0.95(0.66-1.40)$ & $0.93(0.63-1.39)$ \\
\hline T3 & $0.72(0.47-1.11)$ & $0.83(0.54-1.30)$ \\
\hline$P$ trend & 0.13 & 0.42 \\
\hline \multicolumn{3}{|c|}{ Local snacks-fish and seafoods-high salt foods } \\
\hline $\mathrm{T} 1^{\dagger}$ & 1.00 & 1.00 \\
\hline $\mathrm{T} 2$ & $1.01(0.68-1.48)$ & $1.01(0.68-1.50)$ \\
\hline T3 & $1.30(0.87-1.97)$ & $1.26(0.83-1.93)$ \\
\hline$P$ trend & 0.20 & 0.27 \\
\hline \multicolumn{3}{|l|}{ Fruits-legumes } \\
\hline $\mathrm{T} 1^{\dagger}$ & 1.00 & 1.00 \\
\hline $\mathrm{T} 2$ & $0.78(0.52-1.17)$ & $0.85(0.58-1.26)$ \\
\hline T3 & $0.66(0.41-1.07)$ & $0.72(0.48-1.10)$ \\
\hline$P$ trend & 0.08 & 0.13 \\
\hline \multicolumn{3}{|c|}{ Tropical fruits-oats } \\
\hline $\mathrm{T} 1^{\dagger}$ & 1.00 & 1.00 \\
\hline $\mathrm{T} 2$ & $1.35(0.92-1.96)$ & $1.29(0.88-1.90)$ \\
\hline T3 & $1.59(1.08-2.32)$ & $1.30(0.87-1.93)$ \\
\hline$P$ trend & $0.02 *$ & 0.19 \\
\hline
\end{tabular}

AOR, Adjusted odd ratio; CI, Confidence interval; T, Tertile

${ }^{\dagger} \mathrm{T} 1, \mathrm{~T} 2$ and $\mathrm{T} 3$ represent individuals in the lowest, intermediate and highest category of the dietary factor score

${ }^{\ddagger}$ Model 1 adjusted for age, gender, race, energy intake, BMI, marital status and smoking status $\S$ Model 2 further adjusted for education level

${ }^{*} P<0.05$

\section{DISCUSSION}

In this analysis, we have used PCA instead of cluster analysis in deriving DP because it is a more "pragmatic choice" and provides a continuous score for each subject in all components (Crozier et al., 2006). Furthermore, the score can be categorised as tertile, quartile or quintile to observe the conformity of the subjects with DPs and its association with SA. Using the a posteriori DP approach, five DPs have successfully been identified based on the regular food intake of 579 multi-ethnic elderly Malaysians. These are (1) sweet foods-beverages (2) meatvegetables-rice and noodles, (3) local snacks-fish and seafood-high salt foods, (4) fruits-legumes, and (5) tropical fruitsoats. Two models were used to represent 'tropical fruits-oats' DP, which was found to be associated with SA, rather than UA and MCI.

The findings showed that the consumption of tropical fruits was associated with SA among those with a high level of education, which is defined here as receiving formal education up to secondary school and above. This finding was in line with other studies that reported the benefits of fruits consumption with healthy longevity. For example, a longitudinal study by Hodge 
Table 4. Characteristics of subjects with successful aging dietary patterns, by tertiles

Tropical fruits-oats $D P$

(Present as mean \pm SD and \%)
Tropical fruits-oats dietary pattern $\quad$-value

\begin{tabular}{|c|c|c|c|c|c|}
\hline & $n(\%)$ & $T 1^{\dagger}$ & $T 2^{+}$ & $T 3^{\dagger}$ & \\
\hline Age (years) & $68.2 \pm 5.4$ & $68.2 \pm 5.5$ & $68.4 \pm 5.4$ & $67.9 \pm 5.4$ & 0.66 \\
\hline Energy intakes (kcal) & $1648 \pm 411$ & $1735 \pm 418$ & $1597 \pm 392$ & $1611 \pm 408$ & $0.00 * *$ \\
\hline Body mass index & $24.9 \pm 4.2$ & $25.1 \pm 4.0$ & $24.9 \pm 4.2$ & $24.7 \pm 4.3$ & 0.52 \\
\hline Male & $321(55.4)$ & 36.4 & 30.8 & 32.7 & \\
\hline Female & $258(44.6)$ & 29.5 & 36.4 & 34.1 & \\
\hline Race & & & & & 0.05 \\
\hline Malay & $345(59.6)$ & 35.1 & 33.6 & 31.3 & \\
\hline Chinese & $213(36.8)$ & 32.9 & 33.3 & 33.8 & \\
\hline Indian and others & 21 (3.6) & 9.5 & 28.6 & 61.9 & \\
\hline Marital status & & & & & 0.13 \\
\hline Married & $432(74.6)$ & 34.3 & 31.0 & 34.7 & \\
\hline Not married/Widowed & $147(25.4)$ & 30.6 & 40.1 & 29.3 & \\
\hline Smoking status & & & & & $0.00 * *$ \\
\hline Yes & $114(19.7)$ & 50.9 & 28.1 & 21.1 & \\
\hline No & $383(66.1)$ & 28.5 & 33.4 & 38.1 & \\
\hline Ex-smoker & $82(14.2)$ & 31.7 & 40.2 & 28.0 & \\
\hline Education level ${ }^{\S}$ & & & & & $0.04 *$ \\
\hline Low education & $412(71.2)$ & 36.7 & 34.7 & 28.6 & \\
\hline High education & $167(28.8)$ & 25.1 & 29.9 & 44.9 & \\
\hline
\end{tabular}

T, tertile

${ }^{\dagger} \mathrm{T} 1, \mathrm{~T} 2$ and $\mathrm{T} 3$ represent individuals in the lowest, intermediate and highest category of the dietary factor score

*Values reportes as mean $\pm \mathrm{SD}$

\$Low education (no formal education and primary school), High education (secondary school and college)

${ }^{*} p<0.01,{ }^{* *} P<0.001$

et al. (2014) reported that higher intake of fruits together with a lower consumption of meat-based and other fatty food contributed to SA. Other than that, among older Chinese women in Hong Kong, the risk of developing cognitive impairment was also reduced with a DP consisting of a high intake of 'vegetables and fruits' and 'snacks, drinks and milk products' (Chan et al., 2013). However, previous studies incorporated all fruits into one group as compared to our study that found only tropical fruits in the DP component contributed to SA but not the non-tropical fruits. Tropical fruits are rich in natural antioxidants such as vitamins $\mathrm{C}$ and $\mathrm{E}$, polyphenols and carotenoids. The antioxidant properties of selected tropical fruits were studied by Lim, Lim \& Tee (2007) who found that guavas, star fruits and papayas have high primary antioxidant potential compared to oranges. Bananas contain powerful secondary antioxidants. Antioxidant compounds act as scavengers to remove and reform the oxidized biomolecules (free radicals) (Ruszkiewicz \& Albrecht, $2015)$ to protect cognitive functions (Letenneur et al., 2007), thus promoting healthy aging and longevity. The occurrence of cognitive impairment increased with the reduction in intake 
of total dietary fibre and soluble dietary fibre (Lee et al., 2001). On the other hand, individuals with better cognitive function might have a better diet quality with higher carbohydrate and fibre intake including fruits, vegetables, vitamins and minerals (Rue et al., 1997). A longitudinal assessment of protective role of a specific DP is needed to better outline the cause-effect mechanism.

This study also showed that oats contribute to SA. A DP that consists of dairy, cereals and eggs, has been shown to prevent cognitive decline among elderly. A previous study had defined the cereal group as whole grains and refined grains including oats (Ashby-Mitchell, Peeters \& Anstey, 2015). Oats contain antioxidant components such as avenanthramides, tocopherols, tocotrienols, $\beta$-glucan, and phenolic compounds. Avenanthramide, which can be found abundantly in oats, blocks nucler kappa factor B (NF$\kappa B)$ activities and leads to the decrease of inflammation-related cytokine production (Sur et al., 2008). Thus, Lee et al. (2015) recommended that grains, which are high in antioxidants, have a positive role to play in preventing oxidative stress and inflammationrelated chronic diseases. The findings of the study challenge the typical dietary habits of the local population, where rice is the staple food. The white rice, noodles and coffee DP has been identified by Kim et al. (2015) to be associated with lower cognitive function after adjusting for age, sex, educational level, alcohol intake, exercise and diabetes. However, current findings did not find any association between meat-vegetables-rice and noodles group with SA.

Education is one of the important confounders in the association between nutrition and cognition. In model 1, 'tropical fruits-oats' DPs were significantly associated with SA. When educational level was adjusted in model 2 , the association between this DP and
SA was not significant. A similar study by Akbaraly et al. (2009) found that after an adjustment for education in the studied models, the negative correlation between the 'whole food' pattern and the positive correlation between the 'processed food' pattern with cognitive decline were attenuated. Parrott et al. (2013) suggested that among individuals with an equivalent diet quality, the magnitude and characteristics of the diet-cognition relationship depended on their socioeconomic circumstances. The study found that adherence to the Western dietary pattern correlated positively with cognitive decline only among in those with low education. In the present, study 'tropical fruits-oats' DP correlated positively-with SA among those with high education. There is a need to further investigate the challenges faced by those with low education to age successfully.

One of the strengths of this study was that successful agers were compared with two other aging groups against which were UA and MCI rather than only one group such as MCI, as frequently done in other studies. On the other hand, this study used a comprehensive definition of SA as the outcome rather than looking into one element such as cognitive or health status. However, it should be noted that this was a crosssectional comparative study, where the cause and effect cannot be elucidated. We also suggest identifying DP using other statistical methods such as cluster analysis or reduced regression rank, as each method would provide answers to different questions. There is a need to examine the effect of DP on the occurrence of successful aging in a longitudinal study design, with a larger sample size, and with a focus on the challenges faced by the low education group. Further assessment of biomarkers of DP should be conducted using novel method such as the metabolomic approach. 


\section{CONCLUSION}

Principal component analysis allowed the identification of DPs based on data from habitual food intake. We have shown that 'tropical fruits-oats' DP increased the chances of SA by 2.4 times, as compared to UA and $\mathrm{MCI}$, among those with secondary education and above. Promoting a healthy DP such as 'tropical fruits-oats' DP, is a cost-effective strategy to achieve healthy longevity.

\section{List of Abbreviations}

SA: Successful aging; MCI: Mild cognitive impairment; UA: Usual aging; DP: Dietary pattern; DASH: Dietary Approach to Stop Hypertension; ADL: Activities of Daily Living; IADL: Instrumental Activities of Daily Living; MMSE: Mini Mental State Examination; GDS-15: Geriatric Depression Score-15; RAVLT: Rey Auditory Verbal Learning Test; DHQ: Diet History Questionnaire; SPSS: Statistical Package for the Social Sciences; BTS: Bartlett test of sphericity; KMO: Kaiser-MeyerOlkin; PCA: Principal component analysis; BMI: Body mass index

\section{Acknowledgments}

We would like to extend our gratitude to the Ministry of Higher Education for grant LRGS/ $\mathrm{BU} / 2012 / \mathrm{UKM}-\mathrm{UKM} / \mathrm{K} / 01$. We wish to thank all the enumerators and respondents of this study for their cooperation and patience.

\section{Authors' contributions}

NNINMF, conceived and designed the study, performed the experiments, analysed the data and wrote the paper; SS, conceived and designed the study, contributed expertise and provided expert advice on dietary intake; RR, contributed expertise and provided expert advice on dietary intake; MAO, contributed expertise and expert advice on statistical analysis; NCD, contributed expertise and provided expert advice on aging groups criteria; $\mathrm{RR}$, contributed expertise and provided expert advice on aging groups criteria; $\mathrm{SH}$, contributed expertise and provided expert advice on dietary intake; HJJM contributed expertise and provided expert advice on dietary intake; BNAH, performed the experiments and analysed the data; all authors critically reviewed the papers and approved its submitted version.

\section{Conflicts of interest}

The authors declare that they have no conflict of interest. The funding sponsors had no role in the design of the study; in the collection, analyses, or interpretation of data, in the writing of the manuscript, and in the decision to publish the results.

\section{References}

Akbaraly TN, Singh-Manoux A, Marmot MG \& Brunner EJ (2009). Education attenuates the association between dietary patterns and cognition. Dement Geriatr Cogn Disord 27(2):147-154.

Ashby-Mitchell K, Peeters A \& Anstey KJ (2015). Role of Dietary Pattern Analysis in Determining Cognitive Status in Elderly Australian Adults. Nutrients 7(2):1052-1067.

Bowling A \& Dieppe P (2005). What Is Successful Ageing and Who Should Define It? BMJ 331(7531):1548-1551.

Chan R, Chan D \& Woo J (2013). A Cross Sectional Study to Examine the Association between Dietary Patterns and Cognitive Impairment in Older Chinese People in Hong Kong. J Nutr Health Aging 17(9):757-765.

Crozier SR, Robinson SM, Borland SE \& Inskip HM (2006). Dietary patterns in the Southampton Women's Survey. Eur J Clin Nutr 60:13911399.

Fakhruddin NNI, Shahar S, Abd Aziz NA, Yahya HM \& Rajikan R (2016). Which Aging Group Prone to Have Inadequate Nutrient Intake?: TUA Study. Sains Malaysiana 45(9):13811391.

Freeland-Graves J \& Nitzke S (2002). Position of the American Dietetic Association: total diet approach to communicating food and nutrition information. J Am Diet Assoc 107(7):12241232.

Gopinath B, Russell J, Kifley A, Flood VM \& Mitchell P (2016). Adherence to Dietary Guidelines and Successful Aging over 10 Years. J Gerontol A Biol Sci Med Sci 71(3):349-355.

Hodge AM, O'dea K, English DR, Giles GG \& Flicker L (2014). Dietary Patterns as Predictors of Successful Ageing. $J$ Nutr Health Aging 18(3):221-227.

Hu FB (2002). Dietary Pattern Analysis: A New Direction in Nutritional Epidemiology. Curr Opin Lipidol 13(1):3-9.

Imamura F \& Jacques PF (2011). Invited commentary: dietary pattern analysis. Am $J$ Epidemiol 173(10):1105-1108.

Katz S, Downs TD, Cash HR \& Grotz RC (1970). Progress in Development of the Index of ADL. Gerontologist 10(1):20-30. 
Kim J, Yu A, Choi BY, Nam JH, Kim MK, Oh DH \& Yang YJ (2015). Dietary patterns derived by cluster analysis are associated with cognitive function among Korean older adults. Nutrients $7(6): 4154-4169$.

Lawton MP \& Brody EM (1969). Assessment of Older People: Self-Maintaining and Instrumental Activities of Daily Living. Gerontologist 9(3):179186.

Lee L, Kang SA, Lee HO, Lee BH, Park JS, Kim JH, Jung IK, Park YJ \& Lee JE (2001). Relationships between dietary intake and cognitive function level in Korean elderly people. Public Health 115:133-138.

Lee YM, Han SI, Song BC \& Yeum KJ (2015). Bioactives in Commonly Consumed Cereal Grains: Implications for Oxidative Stress and Inflammation. J Med Food 18(11):1179-1186.

Letenneur L, Proust-Lima C, Le Gouge A, Dartigues JF \& Barberger-Gateau P (2007). Flavonoid Intake and Cognitive Decline over a 10-Year Period. Am J Epidemiol 165(12):1364-1371.

Lim Y, Lim T \& Tee J (2007). Antioxidant Properties of Several Tropical Fruits: A Comparative Study. Food Chemistry 103(3):1003-1008.

Parrott MD, Shatenstein B, Ferland G, Payette H, Morais JA, Belleville S, Kergoat MJ, Gaudreau P \& Greenwood CE (2013). Relationship between Diet Quality and Cognition Depends on Socioeconomic Position in Healthy Older Adults. J Nutr 143(11):1767-1773.

Petersen RC, Caracciolo B, Brayne C, Gauthier S, Jelic V \& Fratiglioni L (2014). Mild cognitive impairment: a concept in evolution. J Intern Med ] 275(3):214-228.

Razali R, Jean-Li L, Jaffar A, Ahmad M, Shah SA, Ibrahim N, Din NC, Nik Jaafar NR, Midin M, Sidi H \& Ahmad S (2014). Is the Bahasa Malaysia Version of the Montreal Cognitive Assessment (MOCA-Bm) a Better Instrument Than the Malay Version of the Mini Mental State Examination (M-MMSE) in Screening for Mild Cognitive Impairment (MCI) in the Elderly? Compr Psychiatry 55 (Suppl 1):S70-75.
Roman-Viñas B \& Serra-Majem L (2014). Diet and healthy patterns in the elderly. Curr Nutr Rep 3(2):69-87.

Rue AL, Koehler KM, Wayne SJ, Chiulli SJ, Haaland KY \& Garry PJ (1997). Nutritional status and cognitive functioning in a normally aging sample: a 6-y reassessment. Am J Clin Nutr 65:20-29.

Ruszkiewicz J \& Albrecht J (2015). Changes in the Mitochondrial Antioxidant Systems in Neurodegenerative Diseases and Acute Brain Disorders. Neurochem Int 88:66-72.

Schmidt M (1996). Rey Auditory Verbal Learning Test: Ravlt: A Handbook. Western Psychological Services.

Shahar S, Omar A, Vanoh D, Hamid TA, Mukari SZ, Din NC, Rajab NF, Mohammed Z, Ibrahim R, Loo WH, Meramat A, Kamaruddin MZ, Bagat MF \& Razali R (2016). Approaches in Methodology for Population-Based Longitudinal Study on Neuroprotective Model for Healthy Longevity (TUA) among Malaysian Older Adults. Aging Clin Exp Res 28(6): 1089-1104.

Sur R, Nigam A, Grote D, Liebel F \& Southall MD (2008). Avenanthramides, Polyphenols from Oats, Exhibit Anti-Inflammatory and Anti-Itch Activity. Arch Dermatol Res 300(10):569-574.

Vanoh D, Shahar S, Din NC, Omar A, Vyrn CA, Razali R, Ibrahim R \& Hamid TA (2017). Predictors of poor cognitive status among older Malaysian adults: baseline findings from the LRGS TUA cohort study. Aging Clin Exp Res 29(2):173-182.

Wechsler D (1958). The measurement and appraisal of adult intelligence. The Williams \& Wilkins Company, Baltimore.

Yesavage JA, Brink TL, Rose TL, Lum O, Huang V, Adey M \& Leirer VO (1982). Development and Validation of a Geriatric Depression Screening Scale: A Preliminary Report. J Psychiatr Res 17(1):37-49. 